\title{
LÍMITE NATURAL DE DEUDA PARA LA ECONOMÍA COSTARRICENSE
}

\section{NATURAL DEBT LIMIT FOR THE COSTA RICAN ECONOMY}

\section{Carlos Chaverri Morales ${ }^{1}$}

\begin{abstract}
Resumen
Un indicador complementario a las herramientas tradicionales para el análisis de la sostenibilidad de la deuda es el "Límite Natural de Deuda", (LND) propuesto por Mendoza y Oviedo (2004). Este límite determina el valor de la deuda como proporción del PIB que es coherente con un compromiso por parte del gobierno de mantenerse solvente en caso de que experimente, de forma permanente, una serie de eventos que provoque que los ingresos y gastos se ajusten a niveles mínimos históricos.
\end{abstract}

Utilizando información estadística del Gobierno Central de Costa Rica con frecuencia anual y para el periodo comprendido entre 1985 y 2015, se estima que el valor del LND para la economía costarricense es de 48,6\%.

A la luz de los resultados obtenidos y como parte de la discusión que este documento pretende fomentar, es relevante mencionar que la ausencia de reformas fiscales tendientes a incrementar los ingresos o reducir el gasto público provocaría que en el corto plazo la deuda del gobierno sobrepase el valor del LND. Más aún, un ajuste del gasto público sin mejoras en los ingresos tributarios tan solo retrasaría la convergencia del nivel actual de deuda al umbral definido.

Lo anterior condicionaría el accionar de la política monetaria para cumplir con su objetivo primario de estabilidad de precios.

Doi: http://dx.doi.org/10.15359/eys.22-51.4

Fecha de recepción: 19-09-2016. Fechas de reenvíos: 26-09-2016, 08-11-2016, 09-12-2016, 26-01-2017. Aceptado el 13-02-2017. Publicado el 01-05-2017.

${ }^{1}$ Economista, Departamento de Investigación Económica, Banco Central de Costa Rica (BCCR). Costa Rica. Correo electrónico chaverrimc@bccr.fi.cr. Las ideas expresadas en este documento son del autor y no necesariamente representan las del BCCR.

Carlos Chaverri Morales

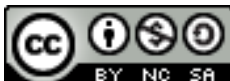

Revista Economía y Sociedad by Universidad Nacional is licensed under a CreativeCommons Reconocimiento-NoComercial- 
Palabras claves: política fiscal; deuda; gestión de la deuda

\begin{abstract}
The "Natural Debt Limit" (NDL) proposed by Mendoza and Oviedo (2004) is a complementary tool to the traditional indicators used to analyze debt sustainability. This limit determines the value of debt as a ratio of GDP, which is consistent with the government's commitment to remain solvent in case the country permanently experiences a series of events that make income and expenses adjust to historical minimum levels.
\end{abstract}

Using annual statistical data from the Central Government of Costa Rica for the 1985-2015 period, the NDL value for the Costa Rican economy has been estimated at $48.6 \%$.

Based on the results obtained and as part of the discussion intended with this paper, it is relevant to mention that the lack of fiscal reforms needed to increase revenue or reduce public spending would make government debt exceed the NDL value in the short term. Moreover, adjusting public spending without improving tax revenues would only delay converging the current level of debt to the defined threshold.

This could limit the actions of the monetary policy to fulfill its primary objective of price stability.

Keywords: fiscal policy; debt; debt management

\title{
1. Introducción
}

Para el Banco Central de Costa Rica, que tiene como objetivo mantener la estabilidad macroeconómica, uno de los principales factores de riesgo es el déficit fiscal y el correspondiente crecimiento de la deuda pública. Por ello, resulta de interés medir con criterios técnicos la sostenibilidad fiscal del país.

Adicionalmente, como lo reconoce la literatura económica, la política monetaria puede enfrentar serias limitaciones para controlar la inflación, en un entorno donde la situación fiscal es insostenible (Sarget y Wallace, 1981) ${ }^{2}$. La literatura sobre el tema destaca que el análisis de la sostenibilidad fiscal es importante debido a los efectos que esta tiene sobre la estabilidad

\footnotetext{
2 Presencia de dominancia fiscal.
}

2

Carlos Chaverri Morales

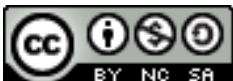

Revista Economía y Sociedad by Universidad Nacional is licensed under a CreativeCommons Reconocimiento-NoComercial- 
macroeconómica, principalmente sobre el desempeño del crecimiento económico, la inflación y la confianza de los mercados internacionales (Reinhart y Rogoff, 2010).

Entre los años 1985-2015, la razón deuda a PIB del Gobierno Central de Costa Rica fue en promedio 31,8\%; a partir de 2008 y coincidiendo con las medidas fiscales tendientes a atenuar los efectos de la crisis financiera de 2007, el nivel de deuda pasó de $24,7 \%$ a $41,4 \%$ hacia finales de 2015, esto es 9,6 p.p. por encima del promedio citado. Dado lo anterior, el objetivo del presente trabajo es estimar el Límite Natural de Deuda (LND) para Costa Rica, como complemento de los indicadores tradicionales utilizados para el análisis de la situación fiscal del país.

El indicador de LND se define como el nivel máximo de deuda que un gobierno es capaz de pagar, aún en un escenario en el cual los ingresos y gastos se ajustan a niveles mínimos observados. Constituye una guía relevante para el análisis de la dinámica y sostenibilidad de la deuda, ya que, a partir de la estimación del límite natural, se puede comparar el nivel observado y, con ello, llevar a cabo análisis de sostenibilidad para horizontes de mediano y largo plazo.

Los resultados obtenidos se utilizan para analizar la sostenibilidad de la deuda bajo dos escenarios: el primero, con base en las recomendaciones de ajuste fiscal hechas por el Fondo Monetario Internacional (en adelante, FMI), en ocasión de la valoración económica del Artículo IV del 2015; y el segundo, tomando como referencia la trayectoria de la deuda, en caso de que se apruebe una reforma fiscal parcial.

El trabajo está estructurado de la siguiente forma, luego de esta introducción, en la sección segunda se lleva a cabo una breve reseña de la evolución de la deuda del Gobierno Central de Costa Rica; en la tercera sección se presenta el enfoque metodológico utilizado y una recopilación de resultados previos, la cuarta sección contiene los resultados obtenidos y la quinta contiene las principales conclusiones.

\section{Evolución de la deuda del Gobierno Central de Costa Rica 1985-2015}

De acuerdo con Rojas (2007), “... en términos generales la evolución de la deuda pública a través del tiempo refleja no solo el impacto de las necesidades de financiamiento del déficit financiero del Sector Público Global, sino también de los cambios en la composición de activos y pasivos financieros del sector público" (p. 6).

Entre los años 1985-2015 la razón deuda a PIB del Gobierno Central fue en promedio 31,8\%; a partir de 2008 y coincidiendo con las medidas fiscales tendientes a atenuar los efectos de la crisis financiera de 2007, el nivel de deuda pasó de $24,7 \%$ a $41,4 \%$ hacia finales de 2015 , esto es 9,6

Carlos Chaverri Morales

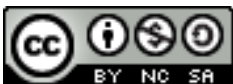

Revista Economía y Sociedad by Universidad Nacional is licensed under a CreativeCommons Reconocimiento-NoComercial- 
p.p. por encima del promedio citado. Adicionalmente la tasa de crecimiento promedio del PIB ${ }^{3}$ fue de $4,6 \%$.

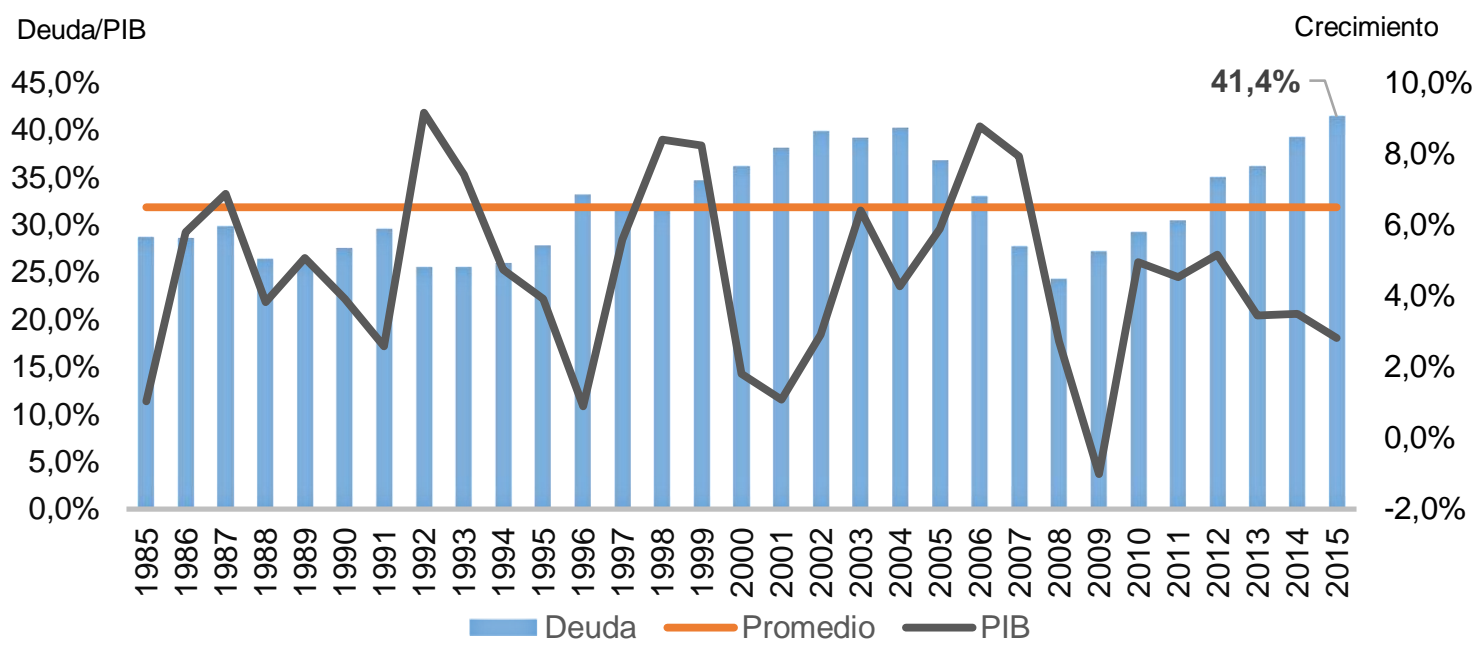

Figura 1. Costa Rica: Deuda del Gobierno Central como porcentaje del PIB y crecimiento del PIB real. 1985-2015. Fuente: elaboración propia con datos del BCCR.

Como se puede apreciar en la figura 1, a partir de 2007 la razón de deuda del Gobierno a PIB registra una tendencia creciente, como resultado de la generación recurrente de déficit primario (desde el 2009), con una tasa real implícita de la deuda superior a la tasa de crecimiento real del producto, particularmente desde el año 2013.

En lo que respecta a la composición de la deuda, esta también ha cambiado a lo largo del tiempo. A mediados de los años ochenta predominaba la denominación en moneda extranjera $(57,9 \%)$ y tan solo un 42,1\% estaba en moneda nacional, mientras que las cifras al 2015 indican que un 75,5\% de la deuda del Gobierno Central está expresada en moneda nacional (Figura 2).

Aunado a lo anterior y particularmente relacionado con la evolución de los ingresos, los gastos y el resultado primario del gobierno, se puede observar el carácter cíclico de los primeros (Tabla 1); en los periodos de aceleración de la actividad económica, los ingresos totales tienden a crecer de forma más rápida, esto es evidente para el periodo comprendido entre 1985 y 2008 . Durante dichos años, la tasa de crecimiento real promedio de la economía fue de $5 \%$ y los ingresos del Gobierno Central crecieron un $21 \%$ en términos nominales (5,8\% en términos reales), con lo cual la proporción ingresos a PIB se mantuvo alrededor del $13 \%$.

\footnotetext{
${ }^{3}$ Basado en la metodología del año base 1991. La tasa de crecimiento potencial es de 4,4\%. 4 Carlos Chaverri Morales

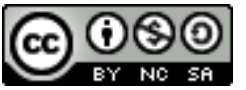

Revista Economía y Sociedad by Universidad Nacional is licensed under a CreativeCommons Reconocimiento-NoComercialCompartirlgual 4.0 Internacional License. 


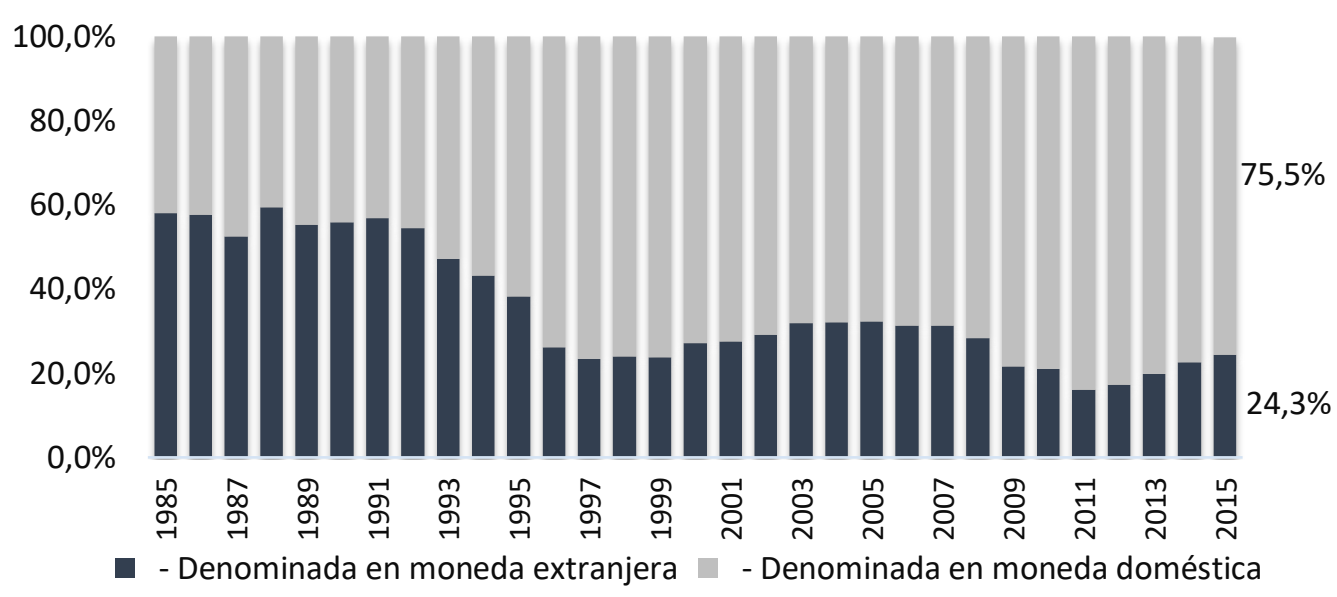

Figura 2. Costa Rica: composición de la deuda del Gobierno Central por moneda (1985-2015). Fuente: elaboración propia.

Luego de la crisis financiera internacional, para el periodo 2009-2015, la actividad económica mermó su ritmo de crecimiento $(3,3 \%)$ y, con ello, convino una desaceleración importante en el crecimiento de los ingresos del gobierno, registrando una variación promedio de $7,2 \%$ en términos nominales (3,2\% en términos reales). Durante este periodo la relación de ingresos a PIB fue de $14,3 \%$.

Tabla 1.

Costa Rica: tasa de crecimiento de los ingresos y gastos del gobierno, crecimiento del PIB y resultado primario como proporción del PIB

\begin{tabular}{|c|c|c|c|c|c|c|}
\hline & \multicolumn{2}{|c|}{ Ingresos } & \multicolumn{2}{|c|}{ Gastos } & \multirow[b]{2}{*}{ PIB } & \multirow{2}{*}{$\begin{array}{c}\text { Resultado } \\
\text { primario }\end{array}$} \\
\hline & Nominales & Reales & Nominales & Reales & & \\
\hline $1985-2008$ & $21,0 \%$ & $5,8 \%$ & $20,3 \%$ & $5,2 \%$ & $5,0 \%$ & $1,0 \%$ \\
\hline 2009-2015 & $7,2 \%$ & $3,2 \%$ & $12,9 \%$ & $8,6 \%$ & $3,3 \%$ & $-2,5 \%$ \\
\hline
\end{tabular}

Fuente: elaboración propia.

Por su parte, los gastos primarios ${ }^{4}$ se pueden analizar en dos etapas, la primera entre los años 1985 y 2008, en la cual el gasto primario como proporción del PIB fue menor en comparación con los ingresos, lo que de cierta forma garantizó alguna sostenibilidad de la deuda e incluso se tradujo en un superávit primario promedio de $1 \%$. La segunda etapa se da a partir de 2005, donde la relación deuda a PIB empieza a crecer más rápidamente que los ingresos, al punto que ya para el año 2009 se genera una brecha (igura 3), provocando a partir de ese año un déficit primario

\footnotetext{
${ }^{4}$ Los gastos primarios se definen como los gastos corrientes menos el pago de intereses y amortizaciones del servicio de la deuda. Se debe tener en cuenta que para el caso costarricense desde hace unos años se han venido incorporado un conjunto de elementos legales e institucionales que han elevado permanentemente el gasto.
} 
en las finanzas del gobierno y aumentando con ello la preocupación acerca del nivel de deuda, dado un gasto creciente y muy rígido, además de pocas fuentes para financiar el déficit del periodo previo con ingresos actuales o futuros.

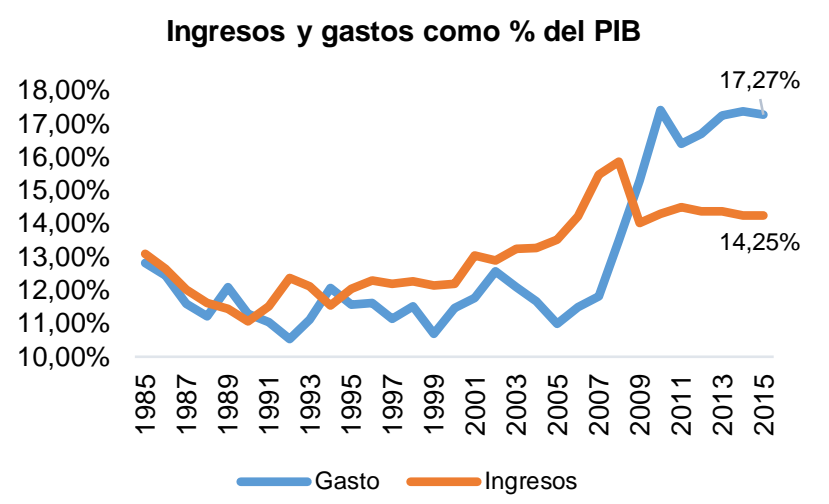

Resultado primario como \% del PIB

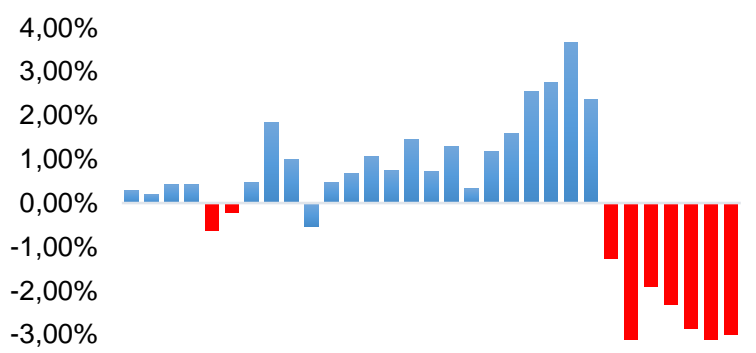

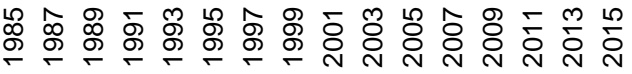

Figura 3. Costa Rica: ingresos, gastos y resultado primario del Gobierno Central como porcentaje del PIB. Fuente: elaboración propia.

Adicionalmente, dada la coyuntura vigente al año 2016 y la incertidumbre sobre la evolución futura de los principales determinantes de la deuda, como lo son el crecimiento económico, la tasa de interés local e internacional y el tipo de cambio, mediante un gráfico de abanico se puede estimar un rango de probabilidad para los posibles valores que la deuda a PIB podría alcanzar en un horizonte de cinco años, a partir de 2016 (Figura 4).

De acuerdo con Boreztein, Cavallo, Cifuentes y Valencia (2013) "la idea del gráfico de abanico es producir una distribución simulada de la deuda respecto al PIB, basado en la dinámica proporcionada por un modelo econométrico de tipo Vector Autoregresivo (VAR) y/o por una serie de pronósticos externos para un grupo de insumos de riesgo que alimentan la ecuación dinámica de la deuda" (p. 31).

Se estima que en ausencia de reformas para mejorar los ingresos y disminuir el gasto, eventos que impliquen una reducción de una desviación estándar ${ }^{5}$ en el crecimiento de la actividad económica o un aumento de una desviación estándar tanto en las tasas de interés local como internacional provocarían que los niveles de deuda respecto al PIB superen el $60 \%$ en el 2020 , con una probabilidad del 29,4\% (Tabla 2).

\footnotetext{
${ }^{5}$ La desviación estándar es una medida del grado de dispersión de los datos con respecto al valor promedio. es una medida de dispersión usada en estadística que nos dice cuánto tienden a alejarse los valores concretos del promedio en una distribución de datos.
}

6

Carlos Chaverri Morales

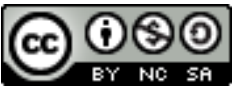

Revista Economía y Sociedad by Universidad Nacional is licensed under a CreativeCommons Reconocimiento-NoComercial- 
Tabla 2.

Costa Rica: probabilidad de superar el nivel actual de deuda como proporción del PIB

\begin{tabular}{|c|c|c|c|c|}
\hline \multicolumn{4}{|c|}{ Razón de (Deuda/PIB) de referencia para 2015 (X) } & \multirow[t]{2}{*}{$41,4 \%$} \\
\hline \multicolumn{4}{|c|}{ Probabilidad } & \\
\hline Año & $x>0.414$ & $X>0.464$ & $x>0.514$ & $x>0.564$ \\
\hline 2016 & $1,8 \%$ & $0,1 \%$ & $0,0 \%$ & $0,0 \%$ \\
\hline 2017 & $16,8 \%$ & $6,3 \%$ & $1,4 \%$ & $0,5 \%$ \\
\hline 2018 & $38,0 \%$ & $22,5 \%$ & $11,3 \%$ & $4,8 \%$ \\
\hline 2019 & $55,5 \%$ & $40,3 \%$ & $27,5 \%$ & $15,7 \%$ \\
\hline 2020 & $69,3 \%$ & $56,0 \%$ & $41,4 \%$ & $28,4 \%$ \\
\hline
\end{tabular}

Fuente: elaboración propia con base en la plantilla "Análisis de la Sostenibilidad Fiscal" del Banco Interamericano de Desarrollo, elaborada por Boreztein, Cavallo et al. (2013).

Tanto el nivel como la probabilidad se incrementarían en la medida en que las valoraciones sobre los riesgos sean mayores ${ }^{6}$.

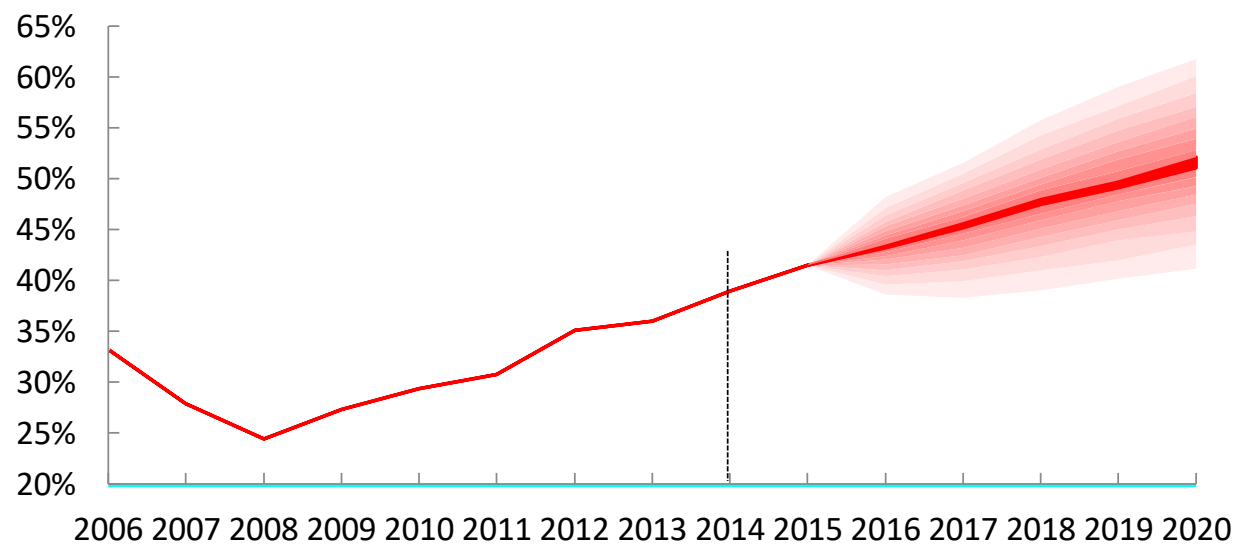

Figura 4. Costa Rica: gráfico de abanico para la trayectoria de deuda a PIB. Fuente: elaboración propia con base en la plantilla "Análisis de la Sostenibilidad Fiscal", del Banco Interamericano de Desarrollo, elaborada por Boreztein, Cavallo et al. (2013).

\section{Metodología utilizada: método de Mendoza y Oviedo}

Como se mencionó en la introducción, el LND determina el porcentaje de deuda a PIB que es coherente con un compromiso por parte del gobierno de mantenerse solvente en caso de que experimente, de forma permanente, una sucesión de eventos, la cual provoque que los ingresos y gastos se ajusten a niveles mínimos.

${ }^{6}$ Los ajustes de las variables exógenas se realizan mediante las desviaciones estándar. 
En otras palabras, este indicador da referencia del valor de deuda sobre la cual el gobierno tiene capacidad de pago. Si el nivel de endeudamiento del gobierno es mayor al LND, este queda sobreexpuesto, ya que en una coyuntura de bajos ingresos fiscales no tendría forma de pagar los compromisos adquiridos.

Dado lo anterior, este indicador puede servir como guía para establecer los mecanismos de acción de mediano y largo plazo, para estabilizar y balancear las finanzas públicas de forma que no se comprometa la capacidad de pago del país. No se debe interpretar como un valor de equilibrio de la deuda ni tampoco como un valor sostenible para una política de endeudamiento.

El punto de partida de la mayoría de modelos existentes para calcular relaciones de deuda a PIB sostenibles es la restricción presupuestaria del gobierno. Esta es una igualdad contable que relaciona todos los flujos de ingresos y pagos del gobierno con los cambios en la deuda pública (ecuación 1):

$$
D_{t+1}=D_{t}\left(1+r_{t}\right)-\left(T_{t}-G_{t}\right)
$$

Donde $D_{t+1}$ es el acervo o saldo de deuda emitida al final del período t; $D_{t}$ corresponde al nivel de deuda sobre la cual el gobierno paga una tasa de interés $r_{t}$ al inicio del periodo $t ; T_{t}$ son los ingresos reales del gobierno y; finalmente, $G_{t}$ son los gastos reales sin el pago de intereses del gobierno. La última expresión de la ecuación (1) define el resultado primario.

Aunado a lo anterior, Mendoza y Oviedo (2004) realizan los siguientes supuestos sobre el desempeño para realizar el cálculo del LND: i) se supone que el crecimiento económico sigue una tendencia determinística ${ }^{7}$ y que crece a una tasa constante $(\dot{y})$, ii) la tasa de interés $r$ es constante, iii) los ingresos públicos son un proceso estocástico exógeno conocido y además el gobierno es averso a sufrir un colapso en sus ingresos; por lo tanto, va a buscar, en la medida de lo posible, que sean estables, a menos de que una eventual pérdida de acceso a los mercados de deuda lo fuerce a ajustar sus gastos a niveles mínimos tolerables. Con base en dichos supuestos, la ecuación (1) puede reescribirse de la siguiente manera:

$$
(1+\dot{y}) d_{t+1}=d_{t}(1+r)-\left(\tau_{t}-g_{t}\right)
$$

En (2) las variables expresadas en letras minúsculas representan relaciones de la variable correspondiente relativa al PIB.

\footnotetext{
${ }^{7}$ Una serie económica tiene una tendencia determinista si conociendo sus valores pasados, se puede determinar sin error sus valores futuros. Con tendencias deterministas no hay incertidumbre sobre los valores futuros.
}

8 
Dado que el gobierno quiere descartar un colapso en sus ingresos por debajo de un mínimo tolerable, no querrá tener un nivel de deuda por encima de aquel al cual no le pueda hacer frente, si el balance primario fuese a mantenerse por siempre en un nivel más bajo o en un estado de crisis fiscal $^{8}$. De esta manera, el LND se aproxima con la siguiente ecuación:

$$
L N D=\frac{\tau^{m i n}-g^{\min }}{r-\dot{y}}
$$

Donde $L N D$ corresponde al valor de referencia de la deuda; $\tau^{\min 9}$ es la mínima recaudación de ingresos como proporción del PIB observada en el período empleado como referencia; $g^{\min }$ corresponde de forma análoga a la menor realización de gasto primario como proporción del PIB. Mientras que en el denominador de la ecuación (3), $r$ representa la tasa de interés real, y finalmente, $\dot{y}$ corresponde a la tasa de crecimiento de estado estacionario de la economía ${ }^{10}$.

La interpretación de la ecuación (3) es la siguiente: si en el periodo $t$, la razón de deuda es menor que el LND y que la realización de la variable estocástica $\tau$ es $\tau^{\min }$, el gobierno financia su razón constante del gasto primario, g, mediante un aumento de la razón de la deuda. Sin embargo, si en el periodo $t$ la razón de la deuda ya es igual al LND y la realización de $\tau$ es $\tau^{\text {min }}$, g debe reducirse a $g^{\min }$.

Los autores señalan que el elemento clave de la política fiscal no es el valor mínimo de la razón de gasto primario en sí mismo, sino la credibilidad del anuncio acerca de un nivel de endeudamiento determinado, el cual depende de la historia del país y de si el proceso probabilístico de los ingresos es endógeno en lugar de exógeno, además de otros factores como lo son: i) la volatilidad de los ingresos tributarios, ii) la flexibilidad de ajuste de los gastos corrientes iii) la tasa de interés real y iv) el crecimiento económico.

\section{Resultados obtenidos}

A continuación se presentan los resultados obtenidos a partir del uso de la metodología de Mendoza y Oviedo (2004) para el cálculo del LND.

\subsection{Estimaciones previas sobre el límite natural de deuda}

En los años recientes y producto de la creciente preocupación acerca de la sostenibilidad de la deuda en Costa Rica, diversos estudios han elaborado estimaciones del LND ubicando dicho valor

\footnotetext{
${ }^{8}$ En relación con este último punto, los autores definen un estado de crisis fiscal como: “la situación en que ocurre una secuencia lo suficientemente larga de la peor realización de ingresos fiscales con un $95 \%$ de confianza y después de que el gasto fiscal se ajusta a su nivel mínimo tolerable con un $95 \%$ de confianza".

${ }^{9}$ Ajustado por la volatilidad del PIB.

${ }^{10}$ Para efectos de esta estimación se utiliza la tasa de crecimiento potencial de la economía costarricense.
} 
en un rango entre $48,8 \%$ y $54,7 \%$. Asimismo, difieren en los resultados básicamente debido al tamaño de la muestra de referencia, la metodología para calcular la tasa de interés real y la forma de eliminar el componente cíclico de los ingresos y del gasto del gobierno, así como del supuesto sobre el crecimiento de la economía en el largo plazo. La Tabla 3 presenta los resultados obtenidos en dichos estudios, así como los supuestos utilizados para su estimación.

Tabla 3.

Costa Rica: estimaciones del límite natural de deuda para la economía costarricense

\begin{tabular}{l|cccccc}
\hline \multicolumn{1}{c}{ Autores } & Lnd & $\begin{array}{c}\text { Periodo de } \\
\text { referencia }\end{array}$ & Ingresos/PIB & Gastos/PIB & $\begin{array}{c}\text { Crecimiento } \\
\mathbf{1 1}\end{array}$ & $\begin{array}{c}\text { Tasa de } \\
\text { interés real }\end{array}$ \\
\hline Espinoza y Valerio (2014) & $54,7 \%$ & $1991-2013$ & $9,2 \%$ & $11,2 \%$ & $3,9 \%$ & $7,5 \%$ \\
Fernández (2005) & $48,8 \%$ & $1990-2000$ & $18,0 \%$ & $14,8 \%$ & $1,8 \%$ & $8,5 \%$ \\
Madrigal, Campo y & $44,4 \%$ & $2008-2013$ & $12,9 \%$ & $12,4 \%$ & $4,4 \%$ & $1,1 \%$ \\
Granados (2014) & & & & & & \\
Mendoza y Oviedo (2009) & $54,5 \%$ & $1990-2005$ & $32,0 \%$ & $29,6 \%$ & $2,0 \%$ & $5,0 \%$ \\
Mendoza y Oviedo (2004) & $53,3 \%$ & $1990-2002$ & $20,2 \%$ & $18,5 \%$ & $1,8 \%$ & $5,0 \%$ \\
\hline
\end{tabular}

Fuente: elaboración propia.

En el presente trabajo se utiliza información anual de las estadísticas fiscales del Gobierno Central para el periodo comprendido entre 1985 y 2015, con ella se ha estimado que el valor del LND para la economía costarricense es de 48,6\%. Dicho valor se aproxima utilizando un $\tau^{\text {min }}$ igual a $10,8 \%$; este valor se obtiene al restarle al promedio de la razón deuda a PIB dos desviaciones estándar para tener un proxy de una mínima realización de ingresos; adicionalmente, se utiliza un $g^{\min }$ igual a 12,8\% que corresponde al promedio observado entre 1985 y 2015.

A criterio de los autores, uno de los temas más sensibles es la estimación de una tasa de interés real para el servicio de la deuda. En los trabajos de referencia de Mendoza y Oviedo (2004) se utiliza una tasa de interés real que se aproxima como una medida de riesgo soberano que resulta del margen entre el índice EMBI+ y la tasa de los bonos del Tesoro de los Estados Unidos, deflactado con el deflactor del PIB.

En el caso costarricense y dada la disponibilidad de información la tasa real, se obtiene a partir de una tasa nominal efectiva transada de la deuda del gobierno, de acuerdo con la siguiente ecuación:

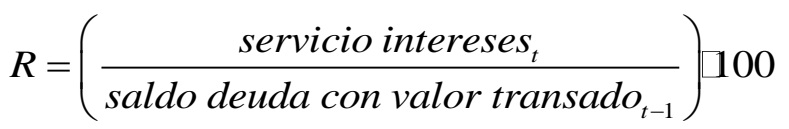

\footnotetext{
${ }^{11}$ Algunas estimaciones utilizan la tasa de crecimiento del PIB per cápita. 10 
La tasa real por definición se obtiene deflactando la tasa nominal $R$ con la inflación medida con el índice de precios al consumidor $(\pi)$ :

$$
r=\frac{(1+R)}{(1+\pi)}-1
$$

Dado lo anterior, se estima una tasa de interés real $(r)$ para el periodo de $0,3 \%$. Mientras que el crecimiento de la economía $(\dot{y})$ es de $4,4 \%$, que corresponde al estimado mediante el filtro de Hodrick y Prescott.

Tabla 4.

Costa Rica: Límite Natural de Deuda

\begin{tabular}{|c|c|}
\hline Deuda Gobierno Central / PIB & $1985-2015$ \\
\hline Promedio & $31,9 \%$ \\
\hline Máximo & $41,4 \%$ \\
\hline Año del máximo & 2015 \\
\hline Ingresos / PIB & $1985-2015$ \\
\hline Promedio & $13,0 \%$ \\
\hline Desviación estándar $(\sigma)$ & 1,3 p.p. \\
\hline Coeficiente de variación & $9,6 \%$ \\
\hline Promedio menos $2 \sigma$ & $10,8 \%$ \\
\hline Nivel mínimo observado en la muestra & $11,0 \%$ \\
\hline Gastos / PIB & $1985-2015$ \\
\hline Promedio & $12,8 \%$ \\
\hline Desviación estándar ( $\sigma)$ & 2,3 p.p. \\
\hline Coeficiente de variación & $17,9 \%$ \\
\hline Nivel mínimo observado en la muestra & $10,5 \%$ \\
\hline Ajuste fiscal implícito & $1,6^{12}$ \\
\hline Objetivo & $1985-2015$ \\
\hline Tasa de crecimiento de la economía & $4,4 \%$ \\
\hline Tasa de interés real & $0,3 \%$ \\
\hline Límite Natural de Deuda & 48,6 \\
\hline
\end{tabular}

Fuente: elaboración propia.

Adicional al LND, y con el fin de permitir una estimación más dinámica, la Tabla 5 contiene diferentes aproximaciones para dicho valor para distintas combinaciones de ajuste en los ingresos y los gastos como proporción del PIB. El ajuste se lleva acabo de acuerdo con la desviación estándar de cada serie; para ambas variables, a partir de la línea de estimación base se suman y

${ }^{12}$ Se refiere al ajuste en términos de desviaciones estándar que se requieren para que el LND sea igual al nivel máximo de deuda observado. 
se restan hasta tres desviaciones estándar, manteniendo constante la tasa de interés real estimada y el crecimiento potencial de la economía.

Tabla 5.

Costa Rica: umbral de LND para diferentes combinaciones de ingreso y gasto como proporción del $P I B$

\begin{tabular}{|c|c|c|c|c|c|c|c|c|c|}
\hline & \multicolumn{7}{|c|}{ Gastos/PIB } \\
\hline & & & \multicolumn{3}{|c|}{ Disminuye } & \multirow[b]{2}{*}{ Base } & \multicolumn{3}{|c|}{ Aumenta } \\
\hline & & & $3 \sigma$ & $2 \sigma$ & $1 \sigma$ & & $1 \sigma$ & $2 \sigma$ & $3 \sigma$ \\
\hline \multicolumn{3}{|c|}{ Ingresos/PIB } & 8,3 & 8,4 & 8,4 & 8,4 & 8,4 & 8,5 & 8,5 \\
\hline \multirow{4}{*}{ Aumenta } & $3 \sigma$ & 10,56 & 51,1 & 50,5 & 50,0 & 49,5 & 48,9 & 48,4 & 47,8 \\
\hline & $2 \sigma$ & 10,55 & 50,8 & 50,2 & 49,7 & 49,2 & 48,6 & 48,1 & 47,5 \\
\hline & $1 \sigma$ & 10,54 & 50,5 & 49,9 & 49,4 & 48,9 & 48,3 & 47,8 & 47,3 \\
\hline & Base & 10,53 & 50,2 & 49,6 & 49,1 & 48,6 & 48,0 & 47,5 & 47,0 \\
\hline \multirow{3}{*}{ Disminuye } & $1 \sigma$ & 10,51 & 49,9 & 49,4 & 48,8 & 48,3 & 47,7 & 47,2 & 46,7 \\
\hline & $2 \sigma$ & 10,50 & 49,6 & 49,1 & 48,5 & 48,0 & 47,5 & 46,9 & 46,4 \\
\hline & $3 \sigma$ & 10,49 & 49,3 & 48,8 & 48,2 & 47,7 & 47,2 & 46,6 & 46,1 \\
\hline
\end{tabular}

Fuente: elaboración propia.

Como es de esperar, disminuciones en el gasto junto con incrementos en los ingresos conllevan a una mayor holgura en la capacidad de endeudamiento; por ejemplo, si los gastos del gobierno disminuyen hasta en tres desviaciones estándar con respecto al promedio base y los ingresos no cambian, el límite natural de deuda sería de 50,2\%. Ese mismo ajuste en el gasto y un aumento de hasta tres desviaciones estándar en los ingresos ubicarían dicho valor en $51,1 \%$. En el caso más extremo, incrementos en el gasto de hasta tres desviaciones estándar, junto con una disminución en los ingresos de la misma magnitud, ubicarían el LND en $46,1 \%$.

Ahora bien, con el resultado base obtenido $(48,6 \%)$ se analizan tres situaciones adicionales: la primera es un ejercicio de simulación de la trayectoria de la deuda si no se llevan a cabo ajustes en los ingresos ni en los gastos; la segunda toma como base las recomendaciones hechas por el FMI (medidas administrativas) ${ }^{13}$; y la tercera es una estimación en la que se supone una reforma fiscal parcial (contención de gasto). Para generar los escenarios de deuda se utiliza el Modelo de Sostenibilidad Fiscal (MSF) del Banco Central de Costa Rica.

Como se puede apreciar en la Tabla 6 , en el escenario base la deuda del gobierno ya estaría superando el LND en 2018 y empezaría a crecer hasta un nivel de 58,5\% en 2020. Los resultados

\footnotetext{
${ }^{13}$ En el caso de la reforma parcial, este escenario contempla lo que en su momento el Ministerio de Hacienda estimó en cuanto a que el rendimiento máximo producto de la puesta en ejecución de los proyectos de reforma tributaria (ingresos y gastos) generan un impacto de hasta 1,35 p.p. en el déficit fiscal, por su parte las estimaciones del FMI en su Artículo IV hablan de 0,4 p.p.
}

12 
de los otros dos escenarios sugieren que, aún con ajustes, el nivel de deuda superará el LND en poco tiempo. En el primer caso, las medidas administrativas no serían suficientes y el LND se estaría superando en 2019 y, en el caso, de la contención de gasto, el límite se estaría superando en 2018.

Tabla 6.

Costa Rica: escenarios de trayectoria de deuda

\begin{tabular}{lcccccc}
\hline & $\mathbf{2 0 1 5}$ & $\mathbf{2 0 1 6}$ & $\mathbf{2 0 1 7}$ & $\mathbf{2 0 1 8}$ & $\mathbf{2 0 1 9}$ & $\mathbf{2 0 2 0}$ \\
\hline Escenario base & $41,4 \%$ & $44,2 \%$ & $\mathbf{4 7 , 4 \%}$ & $51,1 \%$ & $54,7 \%$ & $58,5 \%$ \\
Escenario medidas administrativas & $39,3 \%$ & $42,4 \%$ & $45,2 \%$ & $48,0 \%$ & $\mathbf{4 9 , 1 \%}$ & $49,5 \%$ \\
Escenario contención de gasto & $42,4 \%$ & $45,2 \%$ & $48,0 \%$ & $\mathbf{4 9 , 6 \%}$ & $51,1 \%$ & $52,8 \%$ \\
\hline
\end{tabular}

Fuente: elaboración propia.

\section{Conclusiones}

EI LND establece un límite superior para la deuda pública, pero no constituye per se un nivel de deuda sostenible o un nivel de equilibrio. De acuerdo con los autores, el LND sirve para definir el umbral a partir del cual mayores niveles de endeudamiento no serían coherentes con un compromiso creíble de pago.

Los resultados obtenidos indican que si la deuda del Gobierno Central como proporción del PIB se ubica en valores superiores al 48,6\% (LND), ante choques adversos que afecten las finanzas públicas, la credibilidad en la capacidad del gobierno para mantener su solvencia está comprometida, puesto que los ajustes fiscales requeridos serían muy altos.

Con información disponible para el año 2015 el nivel de endeudamiento del Gobierno Central alcanzó un 41,4\% del PIB y, en ausencia de reformas fiscales para el año 2017, dicho nivel sería de $47,4 \%$, situación que hace más que probable cruzar el LND en el corto plazo.

En la medida en que se siga postergando la aprobación de las reformas fiscales necesarias, mayor será el ajuste requerido para estabilizar la razón de deuda a PIB. Estimaciones recientes por parte del Banco Central indican que, en caso de no tomarse medidas en materia fiscal, el ajuste requerido para estabilizar el crecimiento de la deuda sería de aproximadamente 4,5 p.p. del PIB en el 2020.

Es indudable que la ausencia de un ajuste fiscal introduce riesgos a la estabilidad macroeconómica del país y, particularmente, al accionar de la política monetaria.

En un escenario de dominancia fiscal, los altos niveles de endeudamiento del gobierno pueden comprometer la meta de inflación anunciada por un banco central. 
Un nivel de deuda pública no sostenible se refleja en una importante exposición del sector público al riesgo cambiario (si existe una alta proporción de endeudamiento en moneda extranjera) o en una exposición del sistema financiero al riesgo de mercado, asociado a cambios en la valoración de la deuda pública.

Por otra parte, si los acreedores externos observan que es más probable que la deuda alcance niveles cada vez menos sostenibles exigirán un premio mayor sobre las tasas de interés para los créditos públicos externos, y esto podría afectar incluso el costo del crédito privado a nivel local, lo cual tendría implicaciones sobre el crecimiento económico (inversión y consumo).

Finalmente, si el gobierno debe participar de forma más activa en la captación de fondos prestables se podrían dar presiones al alza en las tasas de interés locales y estrujar las oportunidades de financiamiento del sector privado. En el largo plazo, conllevaría, entre otros, problemas de productividad y acceso a recursos externos que complementen el ahorro local, sin obviar las posibles presiones a la depreciación de la moneda local. Todo ello tiene implicaciones directas sobre el sector real de la economía y, por ende, sobre el bienestar de la sociedad.

\section{Referencias}

Boreztein, E., Cavallo, E., Cifuentes, P. y Valencia, O. (2013). Plantilla Integrada para Análisis de Sostenibilidad de Deuda: Versión 2.0, Versión revisada del manual de instrucciones. Recuperado de http://www.iadb.org/es/investigacion-y-datos/detalles-depublicacion,3169.html?pub id=IDB-TN-576

Espinoza, J. y Valerio, M. (2014). Sostenibilidad Fiscal en Costa Rica, 1991-2013: una aproximación a través del método de Montecarlo. Revista Economía y Sociedad, 19(45), 72-95. doi: http://dx.doi.org/10.15359/eys.19-45.3

Fernández, K. (2005). Evaluación de la Sostenibilidad Fiscal en Costa Rica: Un Enfoque Estocástico (Tesis de Maestría de la Universidad Pontificia Católica de Chile). Recuperado de http://economia.uc.cl/docs/tesis kfernandez.pdf

Madrigal, J., Campo, R. y Granados, L. (2014). Centroamérica: Enfrentando el Reto de la Sostenibilidad de las Finanzas Pública (Documento de Trabajo SECMCA 01-2014). Recuperado del sitio web de la Secretaría Ejecutiva del Consejo Monetario Centroamericano: http://www.secmca.org/INVESTIGACIONES ECONOMICAS/InvestigacionesSECMCA/ $\underline{012014 \text { Sostenibilidad Fiscal.pdf }}$

14 
Mendoza, E. y Oviedo, P. (2004). Public Debt, Fiscal Solvency and Macroeconomic Uncertainty in Latin America: The Cases of Brazil, Colombia, Costa Rica, and Mexico (National Bureau of Economic Research Working Paper No. 10637). Recuperado del sitio National Bureau of Economic Research: http://www.nber.org/papers/w10637.pdf

Mendoza, E. y Oviedo, P. (2009). Public Debt, fiscal solvency and macroeconomic uncertainty in Latin America. The cases of Brazil, Colombia, Costa Rica and México. Economía Mexicana Nueva Época, XVIII(2), 133-173. Recuperado de http://www.economiamexicana.cide.edu/num anteriores/XVIII2/01 EnriqueMendo za (133-173).pdf

Reinhart, C. \& Rogoff, K. (2010). Growth in a time of debt. American Economic Review, 100(2), 573-78. http://scholar.harvard.edu/files/rogoff/files/growth in time debt aer.pdf

Rojas, M. (2007). Deuda y los efectos de la política fiscal: evaluación de vulnerabilidades para la economía costarricense (Documento de Investigación DIE-01-2007-DI). Recuperado del sitio web del Banco Central de Costa Rica: http://www.bccr.fi.cr/investigacioneseconomicas/sectorpublico/Deuda y efectos Politica_Fiscal.pdf

Sargent, T. y Wallace, N. (1981). Some Unpleasant Monetarist Arithmetic. Quarterly Review, (1), 1-17. Recuperado de: https://www.minneapolisfed.org/research/quarterlyreview/some-unpleasant-monetarist-arithmetic 